\title{
Demonstration of Multiple Esterases of the Human Dental Pulp After Electrophoresis in Starch and Acrylamide Gels
}

\author{
DONALD S. STRACHAN, ROBERT RAPP, ${ }^{\circ}$ and JAMES K. AVERY \\ Deparments of Oral Biology and Pedodontics, School of Dentistry and Department of Anatomy, \\ University of Michigan, Medical School, Ann Arbor, Michigan
}

Esterase activity of the dental pulp has been demonstrated by histochemical technics on tissue sections by Burstone (Calcification in Biological Systems, 1960, p. 232) and by Yoshiki (Bull. Tokyo Dent. Coll., 3:14-28, 1962). Aside from the localization of cholinesterases in the human dental pulp by Avery and Rapp (Stain Technol., 33:31-7, 1958) and by Ten Cate and Shelton (Arch. Oral Biol., 11:423-8, 1966), little consideration has been given to the characterization of the type or to the number of esterases that may be present. Using acrylamide gel and electrophoretic technics as described by Davis (Ann. N.Y. Acad. Sci., 121:404$27,1964)$, and staining the gels for esterase activity (B. Friedman, D. S. Strachan, and M. M. Dewey, J. Histochem. Cytochem., 14:560-6, 1966) and for cholinesterase activity (G. B. Koelle and J. S. Friedenwald, Proc. Soc. Exp. Biol. (N.Y.), zo: $617-22,1949)$, it is possible to demonstrate four different esterases from homogenates of human dental pulp.

More than 100 human permanent teeth were used in 25 different experiments. The teeth were opened and the pulps removed and weighed. Four to five pulps were homogenized in 40 percent sucrose (0.01 ml./mg. of pulp tissue), and the equivalent of $15 \mathrm{mg}$. of pulp tissue $(0.15 \mathrm{ml}$. of homogenate) was used as the sample for acryla-

This investigation was supported by U.S.P.H.S. re search grants DE-01604-03 and DE-01923-03 from the National Institute of Dental Research, National In. stitutes of Health, Bethesda, Md.

Additional information is available on request to the authors.

Received for publication February 9, 1967.

* Present address: Department of Pedodontics, School of Dentistry, University of Pittsburgh, Pittsburgh, Pa. mide gel electrophoresis. The electrophoretic separation was for 2 hours at $2 \mathrm{ma}$. per tube at $4^{\circ} \mathrm{C}$. The $\mathrm{pH}$ of the electrode buffer was 8.3 . After staining for 2 to 3 hours, using a-naphthyl acetate and $a$-naphthyl butyrate as substrates, four esterase "bands," or localizations, were shown. Band 2 was more prominent in gels stained with $\alpha$-naphthyl butyrate. Earlier work, using starch gel electrophoresis and the method described by Hunter and Markert (Science, 124:1294-5, 1957), showed only bands 1,2 , and 3 , which had the same characteristics of substrate preference and reaction to inhibitors (diisopropyl fluorophosphate, $10^{-7} \mathrm{M}$, and eserine sulfate, $10^{-1} \mathrm{M}$ ) as bands 1,2 , and 3 shown in acrylamide gels. In starch gels at a $\mathrm{pH}$ of 8.6 , all bands migrated toward the anode. (Band 4 was not demonstrated by starch gel electrophoresis.)

When acetyl- or butyryl-thiocholine was used as substrate, only band 4 was significantly reactive and was sensitive to $10^{-7} \mathrm{M}$ DFP and $10^{-6} \mathrm{M}$ eserine sulfate. The esterase at band 4 was designated as a cholinesterase, considering this preference for choline substrates and the inhibitor profile.

Because of the differences in substrate specificity, electrophoretic mobility, and inhibitor sensitivities, the four pulpal esterases should be identified as individual enzymes and not as isoenzymes or as conformers. The identification of multiple esterases in the human dental pulp is important to the interpretation of the histochemical localization of esterases in tissue sections and in establishing a morphologic and functional role of these enzymes in the metabolism of the pulp.

Currently, studies are being made of the changes in the esterase pattern of rabbit molar pulps after resection of the inferior alveolar nerve.

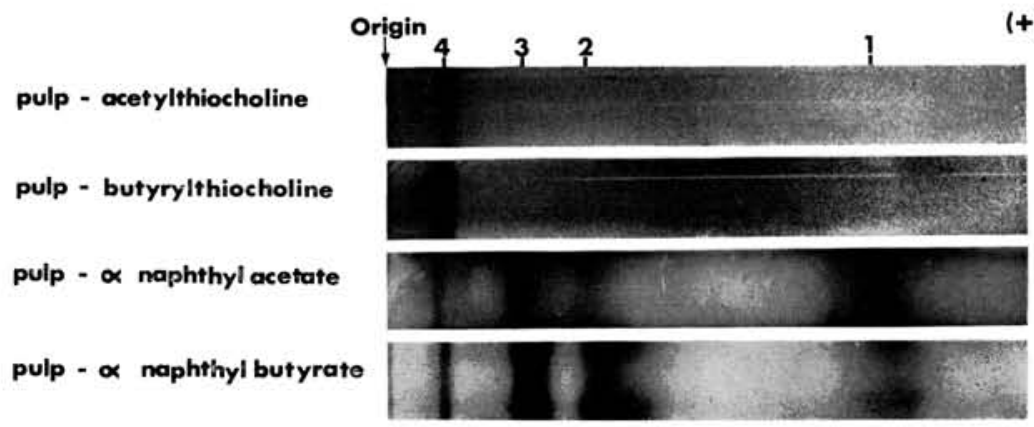

FiG, 1.-Acrylamide gels of human dental pulp stained with substrates noted to left of each gel. The location of the origin is noted and the four bands are labeled $1,2,3$, and 4 . Band 4 is stained with each substrate above and bands 1,2 , and 3 react only with the naphtholic substrates. 